\title{
Contribuições de Pesquisas Brasileiras sobre o Amor e Relacionamentos Amorosos
}

\author{
Adriano Schlösser ${ }^{1}$ \\ Programa de Pós-Graduação em Psicologia da Universidade Federal de Santa Catarina, \\ Florianópolis, Santa Catarina, Brasil \\ Brigido Vizeu Camargo \\ Departamento de Psicologia da Universidade Federal de Santa Catarina, \\ Florianópolis, Santa Catarina, Brasil
}

\begin{abstract}
Resumo
Desde as obras de Freud intituladas "Contribuições à psicologia do amor", a psicologia vem construindo um vasto campo de conhecimento acerca do amor e sobre relacionamentos amorosos. O presente estudo objetivou identificar, por meio de uma revisão sistemática de literatura, os estudos realizados entre os anos de 2002 a 2012 no Brasil, cuja temática se voltasse a relacionamentos amorosos e/ou amor, utilizando-se como palavras-chave os descritores "relacionamentos amorosos" e "amor". Foram consultados os artigos indexados nas bases de dados SciELO, LILACS e PePSIC. Encontraram-se 114 artigos, excluindo-se repetições, publicações estrangeiras ou que não tivessem relação direta com o fenômeno em questão. Os trabalhos nacionais foram classificados de acordo com a temática de estudo e o método utilizado nas suas respectivas publicações. Na categoria temática, os principais temas abordados são: saúde, sexualidade, adolescência, violência, tecnologia, ciúme e psicometria. Na categoria metodológica, foram divididos em: estudos empíricos, estudos teóricos, estudos psicométricos e estudos de caso. Os resultados apontaram um crescimento incipiente de publicações a partir de 2005, bem como uma riqueza de perspectivas teóricas, epistemológicas e metodológicas, o que permite a constatação da fertilidade da temática e sua aplicação em diferentes naturezas, o que não exclui as divergências teóricas que elas mobilizam. Contudo, também se constatou a carência de estudos nacionais, principalmente estudos empíricos de cunho quantitativo e experimental, com predominância em estudos teóricos e da abordagem psicanalítica, e discussões voltadas sobre as relações amorosas na contemporaneidade, em diferentes etapas do ciclo vital.
\end{abstract}

Palavras-chave: Amor, atração interpessoal, revisão.

\section{Contributions of Research on Brazilian about Love and Loving Relationships}

\begin{abstract}
Since the works of Freud entitled "Contributions to the psychology of love", initiated in 1910, psychology has built a vast field of knowledge about love and love relationships. This study aimed to identify, through a systematic literature review, studies conducted between the years 2002 to 2012 in Brazil, whose theme is back to loving relationships and / or love, using as keywords "loving relationships" and "love". Articles have been consulted in SciELO databases, LILACS and PePSIC. About 114 articles
\end{abstract}

Endereço para correspondência: Rua José Antônio Salles, 305, Jardim Iririú, Joinville, SC, Brasil 89224-300. E-mail: adriano.psicologia@yahoo.com.br e brigido.camargo@yahoo.com.br 
were found, excluding repetitions, or foreign publications that do not have direct relationship with the phenomenon in question. The Brazilian studies were classified according to the subject of study and the method used in their respective publications. In thematic category, the main themes are: health, sexuality, adolescence, violence, technology, jealousy and psychometrics. In methodological category, were divided into empirical, theoretical, psychometric studies and case studies. The results showed incipient growth of publications since 2005, as well as a wealth of theoretical perspectives, epistemological and methodological, which allows the realization of the theme of fertility and its application in different natures, which does not exclude the theoretical divergences they mobilize. However it also noted the lack of Brazilian studies, especially empirical studies of a quantitative and experimental, and the predominance of theoretical studies of psychoanalytic approach, with discussions focused on love relationships in contemporary times, in different stages of the life cycle.

Keywords: Love, interpersonal attraction, review.

\section{Contribuciones de las Investigaciones Brasileñas sobre el Amor y las Relaciones Amorosas}

\section{Resumen}

Desde las obras de Freud titulado "Contribuciones a la psicología del amor", la psicología ha construido un vasto campo de conocimiento sobre el amor y las relaciones amorosas. Este estudio tuvo como objetivo identificar, a través de una revisión sistemática de la literatura, los estudios llevados a cabo entre los años 2002 y 2012, a Brasil, cuyo tema sea las relaciones amorosas y el amor. Se consultó a los artículos indexados en las bases de datos SciELO, LILAS y PePSIC. Hemos encontrado 114 artículos, con exclusión de repeticiones o publicaciones extranjeras que no tengan relación directa con el fenómeno en cuestión. Los estudios nacionales se clasificaron de acuerdo con el tema de estudio y el método utilizado en sus respectivas publicaciones. En la categoría temática, los temas principales son: salud; sexualidad; adolescencia; violencia; tecnología; celos y psicometría. En la categoría metodológica, se dividieron en empíricos, estudios psicométricos, teóricos y casos clínicos. Los resultados mostraron un crecimiento incipiente de publicaciones desde el año 2005, así como una gran variedad de perspectivas teóricas, epistemológicas y metodológicas, que permite la verificación de la fertilidad del tema y su aplicación en diferentes naturalezas, que no excluye las divergencias teóricas que movilizan. Sin embargo, también señaló la falta de estudios empíricos nacionales, sobre todo de un enfoque cuantitativo y experimental. Hay predominancia en los estudios teóricos y psicoanalíticos, con las discusiones que se centraron en las relaciones de amor en los tiempos contemporáneos, en las diferentes etapas del ciclo de vida.

Palabras clave: Amor, atracción interpersonal, revisión.

Desde a Idade Antiga, o fenômeno do amor tem sido alvo de interesse e debate dos mais diversos campos de saber, desde o campo filosófico (May, 2012), até a contemporaneidade, mantendo sua vitalidade e importância na vida das pessoas. Neste trajeto, o tema também passou a fazer parte do campo da ciência, após um período de debates e amadurecimento do seu saber.

No campo do conhecimento psicológico, Sigmund Freud foi pioneiro na introdução do amor para a psicologia. Para o criador da psicanálise, o amor se revela quando um indivíduo deseja - ou procura - dar e receber prazer e/ou satisfações a uma outra pessoa, tais como: proteção, admiração, etc. (Doron \& Parot, 1991). Em seus textos intitulados "Contribuições à Psicologia do Amor" (1910/1970, 1912/1970, 1918/1970), Freud atribui ao amor a fonte de importantes vínculos interpessoais, mesmo permeado por dificuldades, sendo que a eleição do objeto de apaixonamento ocorre de forma repe- 
titiva, o que traz à tona a uma perspectiva de determinação inconsciente (Ferrari, 2009).

Nesta perspectiva, o contexto da psicologia enquanto campo científico fez florescer inúmeros estudos e teorias que abarcam a temática do amor, bem como pesquisas em um dos relacionamentos interpessoais no qual o amor mais se enfoca: os relacionamentos amorosos. Os estudos sobre o amor e relacionamentos amorosos passaram a fazer parte do âmbito do campo teórico denominado atração interpessoal (Alferes, 2004), a partir da década de 60 , sendo visto atualmente como fundamental para um relacionamento amoroso de sucesso (Matos, Féres-Carneiro, \& Jablonski, 2005; Sternberg \& Barnes, 1985).

O conceito relativo ao amor, dentro da perspectiva psicológica, é compreendido como um sentimento multidimensional, estrutural e dinâmico (Cassepp-Borges \& Teodoro, 2009; Engel, Olson, \& Patrick, 2002), e não apenas um conceito filosófico ou metafísico, gerando assim um tema de constante interesse para pesquisadores tanto da ciência psicológica quanto de outras ciências sociais (de Almeida, 2012; Cassepp-Borges \& Teodoro, 2007; Hendrick \& Hendrick, 2006; Mosmann, Wagner, \& Féres-Carneiro, 2006; Sternberg \& Weis, 2006; Wachelke, de Andrade, Souza, \& Cruz, 2007). A psicologia do amor é uma ciência que abarca diversas explicações e constructos teóricos - tais como literatura, filosofia, antropologia e a psicologia, tratando de temáticas que envolvem a gênese, manutenção e término das relações amorosas. As diversas teorias que compõem o universo da psicologia propõem olhares diferenciados sobre o amor.

Do ponto de vista da psicologia clínica, o amor pode estar associado a noções de sexualidade sublimada (Freud, 1973), cuidado, responsabilidade, respeito e conhecimento (Fromm, 1956; Schlösser, Dalfovo, \& Delvan, 2012) bem como gratificação de necessidades (Maslow, 1974). Pela perspectiva evolucionista, o amor é concebido como uma consequência de necessidades instintivas sexuais, receber e oferecer proteção e instrumento cognitivo de reprodução (Buss, 2006; Wilson, 1981), enquanto que o behaviorismo compreende o amor como um reforçamento mútuo de comportamentos (Skinner,
1991), posicionamento confirmado atualmente pelos estudos de neuroimagem, que observam que, quando se mostrava a foto da pessoa amada, o sistema de recompensas era o que possuía maior ativação cerebral (Aron et al., 2005). Já a psicometria busca enfocar o caráter estrutural do amor, analisando-o enquanto um conjunto de cognições, afetos, e motivações que necessitam de mensuração, atestanto assim uma objetividade ao fenômeno (de Andrade \& Garcia, 2012; Rubin, 1970; Sternberg, 1986, 1989; Sternberg \& Grajek, 1984).

Para explicar as demandas e variáveis deste complexo fenômeno, a partir da década de 70 desenvolveram-se diversos modelos teóricos sobre o amor no campo científico, bem como instrumentos psicométricos com a finalidade de dar suporte empírico. Ressalta-se aqui a produção de Zick Rubin (1970), precursor da construção de teorias sobre o amor e primeiro teórico ao abordar o amor romântico no campo da psicologia social.

Os principais modelos teóricos sobre o amor na contemporaneidade são: teoria triangular do amor, proposto por Sternberg (1986, 1989, 2000), teoria tipológica do Amor, de Alan John Lee (1977), teoria do apego e o amor romântico (Hazan \& Shaver, 1987), teoria dualista do amor (Berscheid, 2006; Berscheid \& Hatfield, 1969), bem como as teorias de abordagem biológico-evolutivas (Buss, 2006; Kenrick, 2006).

A teoria tipológica do amor, também conhecida por "As Cores do Amor", foi desenvolvida por Lee $(1973,1977)$, propondo uma taxonomia para o amor romântico (de Andrade \& Garcia, 2009). Vale ressaltar o que propõe de Andrade, Sánchez-Aragón e Wachelke (2007, p. 91) em que os estilos de amor são compreendidos fundamentalmente como "... o componente cognitivo individual do fenômeno amoroso". Segundo o autor, assim como existem cores primárias que originam cores secundárias, $\mathrm{o}$ amor também possui estilos primários que, quando combinados, podem originar outros estilos de amor (Lee, 1973).

Posteriormente, Hendrick e Hendrick (2006; Hendrick, Hendrick, \& Dicke, 1988), baseados na teoria de Lee, desenvolvem a Escala de Estilos de Amor (Love Styles Scale). Alguns estu- 
dos foram realizados em diferentes contextos, obtendo resultados parecidos aos de Hendrick e Hendrick (2006; Kanemasa, Taniguchi, \& Daibo, 2004; Sánchez-Aragón, 2005), podendo-se inferir na universalidade dos aspectos pontuados na escala.

Outra teoria bastante citada no corpo científico sobre a temática é a Teoria Triangular do Amor, proposta por Sternberg $(1986,1989)$. Segundo Sternberg e Grajek (1984), o amor é uma das mais intensas e desejáveis emoções humanas, e implica numa combinação de três componentes fundamentais, também chamados de "triângulo do amor", interdependentes entre si: intimidade, paixão e compromisso (Sternberg, 1986), sendo que podem alterar, de acordo com o momento histórico, espaço geográfico e espaço cultural (Cooper \& Pinto, 2008).

No Brasil, um dos estudos pioneiros sobre relacionamentos amorosos foi o de B. F. Reis (1992), no qual realizou uma revisão de literatura acerca de diversas teorias sobre o amor, bem como a dissertação de mestrado de Bystronski (1992) sobre as consequências da liberação dos costumes sobre os relacionamentos amorosos. Ambos autores fazem uso da definição de Rubin (1970), que compreende o fenômeno do amor como uma atitude que é mantida por uma pessoa em relação a uma outra pessoa, predispondo de comportamentos específicos voltados a pessoa amada.

Os estudos sobre relacionamentos amorosos em pesquisas brasileiras têm sido diversificados. No campo da psicometria, instrumentos de medidas de satisfação ou qualidade de relacionamentos românticos foram desenvolvidos, tais como: Escala de Satisfação Conjugal (ESC; M. F. Dela Coleta, 1989), objetivando avaliar a satisfação em áreas da vida conjugal; Escala Fatorial de Satisfação com Relacionamento de Casal (EFS-RC; Wachelke, de Andrade, Cruz, Faggiani, \& Natividade, 2004; Wachelke et al., 2007), visando medir a satisfação do indivíduo com seu relacionamento romântico; e Escala de Avaliação de Qualidade em Relacionamentos Românticos (Aquarela-R; de Andrade \& Garcia, 2012), também com o objetivo de avaliar a qualidade em relacionamentos românticos.
Além disso, estudos sobre propriedades psicométricas e de validação de escalas internacionais também são constatados no cenário nacional, como: estudos sobre a Escala Triangular do Amor de Sternberg - ETAS, inicialmente adaptada por Hernandez (1999) e posteriormente abreviada em uma versão reduzida - ETAS-R, por Gouveia, Fonseca, Cavalcanti, Diniz e Dória (2009; Cassepp-Borges \& Pasquali, 2012; Cassepp-Borges \& Teodoro, 2007, 2009; de Andrade, Garcia, \& Cassepp-Borges, 2013; Mônego \& Teodoro, 2011); validação da versão brasileira da Escala de Estilos de Amor - LAS (de Andrade \& Garcia, 2009); adaptação da Escala Tetrangular do Amor (Gouveia, de Carvalho, Santos, \& de Almeida, 2013; Gouveia et al., 2009); características psicométricas da Relationship Assessment Scale (ReLAS; Cassepp-Borges \& Pasquali, 2011), dentre outros.

Outros estudos empíricos sobre algumas facetas do amor e relacionamentos amorosos foram evidenciados nacionalmente, destacando-se estudos de pesquisadores pioneiros no assunto, como Bystronski $(1992,1995)$, M. F. Dela Coleta (1989, 1990, 1991), e Hernandez (1999; Hernandez \& Biasetto, 2003; Hernandez \& Roveda, 1998). Atualmente, as pesquisas no Brasil também têm centrado suas atividades nos fatores de satisfação e insatisfação do relacionamento conjugal e fatores de ajustamento e desajustamento das relações (de Andrade, Garcia, \& Cano, 2009; Wachelke et al., 2004), relações amorosas e dimensões tecnológicas (A. S. M. Dela Coleta, Dela Coleta, \& Guimarães, 2008), relações amorosas e habilidades sociais (Villa, Del Prette, \& Del Prette, 2007), a relação do amor com as teorias da psicologia social (de Andrade \& Wachelke, 2011; Martins-Silva, Trindade, \& Silva, 2013), dentre outras.

Objetiva-se aqui realizar uma revisão sistemática da produção científica nacional relacionada ao amor e relacionamentos amorosos. Tal esforço vem a contribuir com uma visualização de publicações do Brasil sobre esta temática, elencando temas e métodos utilizados, bem como indicar direcionamentos para pesquisas futuras neste campo teórico. 


\section{Método}

A presente pesquisa documental, de cunho exploratório, realizou uma revisão sistemática da literatura sobre relacionamentos amorosos e amor, mediante a busca em bases de dados, utilizando como fontes de consultas as bases de dados nacionais ScELO, LILACS e PePSIC . Para tanto, foi utilizada uma busca com os descritores "relacionamentos amorosos" e "amor", restringindo os trabalhos voltados ao campo psicológico destes fenômenos. Em todas as bases de dados, foram registrados os artigos publicados entre 2002 a 2012.

Selecionaram-se estudos que adentravam na temática de amor e relacionamentos amorosos, com ligação direta ao campo da psicologia. Inicialmente, buscou-se visualizar os resumos das publicações, para um posterior acesso aos trabalhos completos, sendo os mesmos lidos e analisados de acordo com os critérios de inclusão/exclusão. Os artigos que apenas possuíam resumo como única forma de acesso também foram contabilizados, nos casos em que tivessem relação direta com o fenômeno de relacionamento amoroso.

Por critérios de inclusão, se incluiu apenas artigos indexados, publicados no país no idioma português, dentro do período já citado; e ainda pesquisas quantitativas, qualitativas, revisões de literatura e estudos clínicos. Os critérios de exclusão foram: artigos não indexados, teses, dissertações, resenhas, livros e capítulos de livros. A partir disto, a análise do material foi dividida em quatro fases, conforme ponderam Lakatos e Marconi (2003): (a) apreciação crítica do material; (b) decomposição dos elementos essenciais; (c) agrupamento e classificação; (d) análise final.

As Tabelas 1 e 2 apresentam, sequencialmente, as características das categorias temáticas e metodológicas utilizadas para a classificação dos estudos. As temáticas em relação ao amor e/ ou relacionamento amoroso foram agrupadas e classificadas na Tabela 1, gerando 10 categorias, onde cada uma delas agrupa os estudos relacionados ao fenômeno em questão. Mediante este fato, uma produção poderia ser classificada em mais de um tema de investigação, de acordo com os temas abordados.

Já a Tabela 2 apresenta as definições das categorias metodológicas, evidenciadas no corpo dos artigos. A partir destas definições, a produção foi classificada em um único tema de investigação, a partir dos critérios estipulados.

\section{Tabela 1}

\section{Categorias Temáticas Elaboradas para Classificação dos Estudos}

Categorias Temáticas

Saúde

Estudos cujo foco se referem à questões ligadas à: relação entre amor e/ou relacionamentos amorosos e o processo saúde/doença. Exemplos: depressão; sofrimento psíquico; HIV; tentativas de suicídio e amor patológico.

Sexualidade Produções científicas que trabalham com temáticas voltadas à sexualidade, em diferentes etapas do ciclo vital. Exemplos: relação entre amor e sexo; comportamento sexual; educação sexual; infidelidade; vida afetivo/sexual; bem como valores, crenças, atitudes e representações sociais sobre o sexo.

Psicanálise Artigos que compõem esta área do conhecimento e/ou englobam discussões sobre relacionamentos amorosos ou amor. As temáticas são: amor na obra freudiana; relação entre amor e sexo; psicose; gozo; transferência; resposta psicótica e pulsões.

Juventude Manuscritos que focalizam a temática do amor e relacionamentos amorosos na perspectiva da adolescência e juventude.

Ciúme Artigos que possuam a temática do ciúme no contexto das relações amorosas, como: presença e ausência de ciúme; ciúme romântico; relação entre ciúme e infidelidade e relação entre amor e ciúme.

Relacionamento Produções voltadas aos relacionamentos conjugais hetero e homossexuais de diferentes Conjugal modalidades, como: pegar/ficar; namoro; casamento e recasamento, bem como em diferentes estágios da relação: seleção de parceiros; início, manutenção e término de relacionamentos. 
Instrumentos Psicométricos

Tecnologia

Violência

Outros
Aplicação e validação de escalas; desenvolvimento de medidas psicométricas, ou aspectos teóricos referentes a instrumentos de medidas relativos a amor e/ou relacionamentos amorosos.

Grupo de produções científicas que trouxeram relações entre relacionamentos amorosos no contexto cibernético. Exemplos: sites de relacionamentos; materiais multimídia; vínculos amorosos na Internet e amor virtual.

Estudos que contenham a violência como enfoque, relacionando-se com o amor e/ou relacionamentos amorosos.

Textos que apareceram em baixa quantidade e não puderam ser enquadrados em outras categorias. Exemplos: moralidade; relação entre amor e dinheiro; estudos de cartas de amor; provérbios e na literatura; e amor pós- moderno.

Tabela 2

Categorias Metodológicas Elaboradas para Classificação dos Estudos

Categorias Metodológicas

Estudos

Empíricos

Estudos Teóricos Engloba revisões sistemáticas, contendo revisão de literatura que descreva os procedimentos metodológicos na confecção do artigo, e revisões não-sistemáticas, que não apresentam a descrição dos procedimentos metodológicos.

Estudos de Caso Estudos de fatos particulares, de caráter descritivo e ideográfico.

Estudos Contêm artigos de validação, desenvolvimento e/ou aplicação de escalas voltadas a Psicométricos

Produções científicas com coleta de dados em grupos específicos, mediante a aplicação de questionários, escalas ou entrevistas. Estudos de natureza exploratória, descritiva, correlacional, experimental e quase-experimental, de abordagens tanto quantitativas quanto qualitativas.

relacionamentos amorosos e/ou amor.

\section{Resultados}

A partir da busca feita nas bases de dados utilizadas, foram encontrados 114 artigos, entre os anos 2002 a 2012. Pode ser visualizada, na Figura 1, a frequência na evolução de publicações científicas acerca de relacionamentos amorosos e/ou amor.

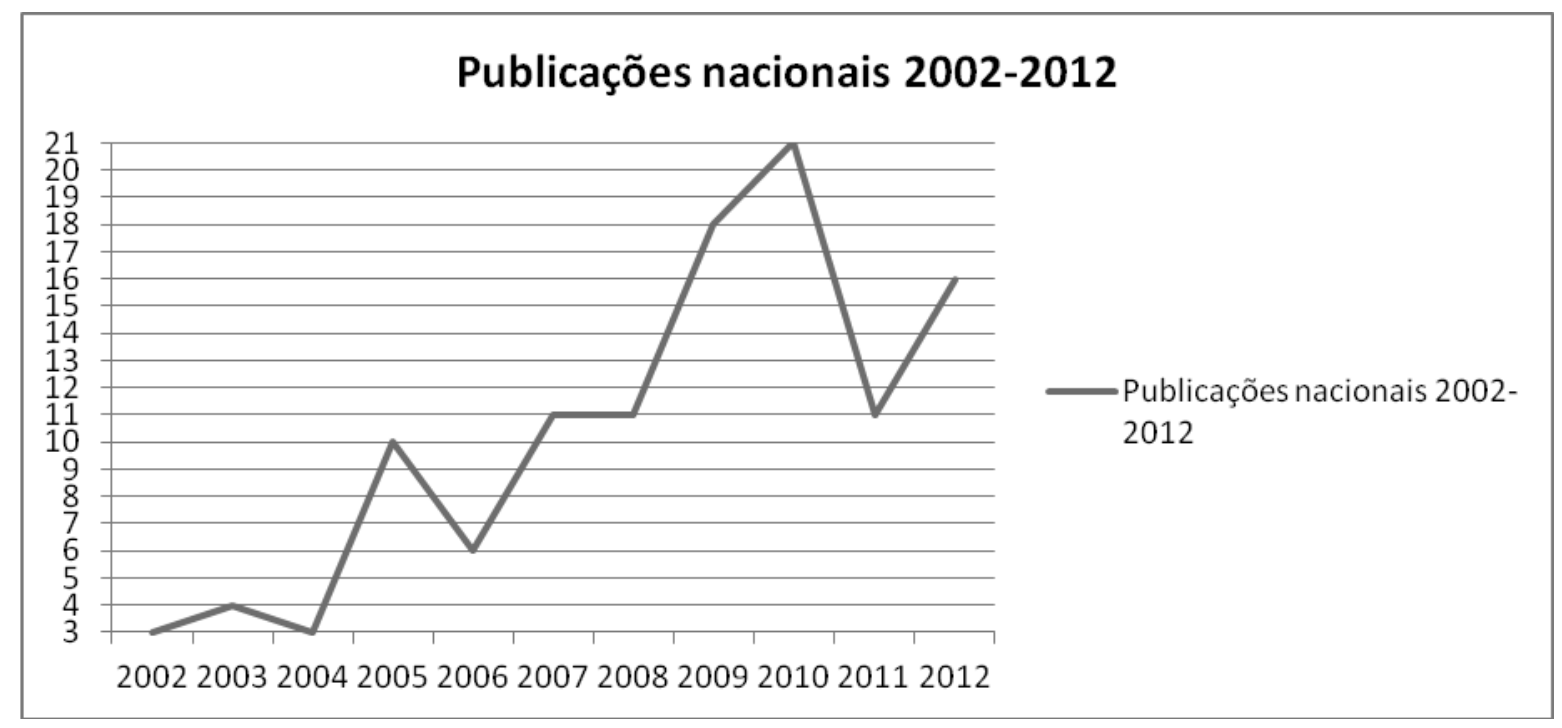

Figura 1. Frequência de publicações científicas nacionais sobre relacionamentos amorosos e/ou amor entre 2002 e 2012. 
Em 2005, observou-se um aumento significativo de produções, distribuindo-se de forma equilibrada ao longo dos anos, chegando a 21 trabalhos no ano de 2010. A linha de crescimento nestas publicações indica uma maior preocupação e interesse de pesquisadores brasileiros sobre a temática, fortalecendo este campo do conhecimento psicológico através de novas contribuições científicas.

Este dado apresenta-se como altamente significativo, do ponto de vista do crescimento científico da psicologia do amor do país, considerando que a temática tem sido fundamentada predominantemente pelo conhecimento leigo, sem rigor teórico-metodológico. Este aumento de publicações pode estar associado a uma maior preocupação dos pesquisadores brasileiros com a temática das relações interpessoais - enfatizando aqui os relacionamentos amorosos - haja vista que são nelas que o ser humano experiência suas emoções e sentimentos mais fortes, tais como o amor.

No panorama nacional, o conhecimento científico sobre amor e relacionamentos românticos proporciona maior solidez empírica frente a fenômenos tão complexos que se relacionam a esta temática, como: satisfação e insatisfação conjugal; escolha de parceiros(as); estabelecimento, manutenção de término de relações românticas; componentes do amor; dentre outros. Ademais, na atual conjuntura de mudanças nas relações amorosas, com novas formas de disposições nos relacionamentos e em papeis sexuais, verifica-se a necessidade de compreender como isso repercute nas relações sociais, influenciando nas ligações afetivas entre pares.

O aumento nas produções também problematiza a necessidade de analisar os relacionamentos amorosos na contemporaneidade, considerando sua importância na felicidade, bem estar e na qualidade de vida da população. Sabe-se que ocorreram modificações nos conceitos relativos ao amor ao longo do tempo (Alferes, 2004; Engel et al., 2002), e o aprimoramento teórico e pesquisa contínua deste fenômeno e construtos relacionados tende a ganhar cada vez mais enfoque, frente às modificações que ocorrem no cenário social. A Tabela 3 apresenta os resultados da quantidade de produções, divididas nas categorias metodológicas e temas de investigação.

Tabela 3

Métodos de Investigação dos Estudos e Temas de Investigação

\begin{tabular}{ccc}
\hline Método de Investigação & Frequência & $n=114$ \\
\hline Estudos Teóricos & 58 & 50,8 \\
Estudos Empíricos & 46 & 40,5 \\
Estudos Psicométricos & 06 & 5,2 \\
Estudos de Caso & 04 & 3,5 \\
Temas de Investigação & Frequência & $\%$ \\
Relacionamento Conjugal & 30 & 26,3 \\
Psicanálise & 29 & 25,4 \\
Sexualidade & 23 & 20,1 \\
Adolescência/Juventude & 17 & 15,0 \\
Saúde & 17 & 15,0 \\
Outros & 11 & 9,6 \\
Violência & 09 & 7,9 \\
Tecnologia & 06 & 5,2 \\
Ciúme & 06 & 5,2 \\
Psicometria & 05 & 4,3 \\
\hline
\end{tabular}


Conforme se observa na Tabela 3 , houve maior predominância de palavras ligadas ao tema "Relacionamento conjugal" $(26,3 \%$ de palavras-chave), sendo que se acoplou nesta categoria produções científicas que retratassem diferentes formas de relacionamentos amorosos. Exemplos desta categoria seriam os estudos de Mosmann, Lomando e Wagner (2010) sobre coesão e adaptabilidade conjugal em homens e mulheres hetero e homossexuais; e o de Duarte e Rocha-Coutinho (2011) sobre o "namorido" como uma nova forma de relacionamento conjugal. Scorsolini-Comin e Santos (2010) fizeram uma importante revisão integrativa da literatura científica nacional acerca da satisfação conjugal, verificando a associação deste construto com a saúde e qualidade de vida. Também se incluiu estudos de diferentes etapas dos relacionamentos, desde a seleção de parceiros (Gianfaldoni et al., 2009) até o término de relacionamentos (Marcondes, Trierweiler, \& Cruz, 2006). Ressalta-se que, nesta categoria, a maior parte dos trabalhos foram de natureza empírica.

Em sequência, a temática "Psicanálise" foi a segunda categoria com maior frequência $(20,1 \%$ das palavras), incluindo nela os artigos que fizeram uso das teorias psicanalíticas para explicar temas pertinentes ao amor e/ou relacionamentos amorosos, sob diferentes perspectivas. De modo geral, a predominância de artigos desta categoria foram estudos teóricos não sistemáticos (Bressaneli \& Teixeira, 2012; Ferrari, 2009; Schlösser et al., 2012).

A "Adolescência" e "Saúde" ocupam simultaneamente a terceira posição, com $15 \%$ cada um. Enquanto os estudos foram classificados na temática adolescência trouxeram temáticas sobre relacionamentos amorosos e/ou amor na perspectiva do adolescente ou jovem, enfocando suas formas de se relacionarem amorosamente ou seus modos de compreensão frente a este fenômeno (de Araújo, 2012; Camargo, Giacomozzi, Wachelke, \& de Aguiar, 2010; Chaves, 2010; Costa \& Fernandes, 2012), os estudos que adentraram na temática saúde enfocaram sobre a influência ou relação dos relacionamentos amorosos na saúde, trazendo entre seus estudos temas sobre AIDS (Manuel, 2009; R. K. Reis \&
Gir, 2010), amor patológico (Sophia, Tavares, \& Ziberman, 2007; Vendrame et al., 2012) ou depressão (Guede, Monteiro-Leitner, \& Machado, 2008).

Estudos nos quais a temática "Violência" se fez presente ocupou $7,9 \%$ do total das publicações, com temática acerca da relação entre amor e violência, violência física e psicológica praticadas no contexto de relacionamentos amorosos, bem como violência de gênero em relacionamentos íntimos (Dias \& Machado, 2011; Levy \& Gomes, 2008; Moura, Lefevre, \& Moura, 2012). Por sua vez, estudos cujas palavras-chave adentraram na categoria "Ciúme" computaram 5,2\%, das publicações, com estudos sobre a relação entre o amor e o ciúme (Baroncelli, 2011), ciúme e infidelidade (de Almeida, 2012), ciúme romântico e violência (Centeville \& de Almeida, 2007), dentre outros.

Ainda, $5,2 \%$ dos artigos foram classificados na categoria "Tecnologia", sendo esta composta em sua maioria por estudos empíricos que trazem correlações entre os relacionamentos amorosos contemporâneos na realidade tecnológica. Como exemplos, apresentam-se os estudos de Donnamaria e Terzis (2009) sobre a busca de parceiro e a evolução de vínculos amorosos na Internet; Bezerra e Justo (2010) que realizaram uma análise de consultas apresentadas em sites de agenciamento amoroso; e A. S. M. Dela Coleta et al. (2008), sobre a possibilidade do relacionamento amoroso via internet.

Também se formou uma categoria com publicações voltadas ao campo psicométrico em território nacional, com validação de escalas e construção de instrumentos de mensuração de relacionamentos amorosos, tais como um estudo nacional dos atributos psicométricos da Escala Triangular do Amor de Sternberg (Cassepp-Borges \& Pasquali, 2012); o desenvolvimento de uma medida multidimensional para avaliação de qualidade em relacionamentos românticos - Aquarela-R (de Andrade \& Garcia, 2012) e validação de escalas para avaliação do amor patológico (Berti et al., 2011). Por fim, outras temáticas foram categorizadas, contudo, pela baixa pela baixa frequência e impossibilidade de categorizar em quadros temáticos, criou- 
se a categoria "Outros", computando $9,6 \%$ de artigos. Exemplos dessa temática são: relação entre amor e dinheiro (Russo, 2011); concepções sobre relações amorosas através de provérbios (Menandro, Rolke, \& Bertollo, 2005); ou estudo com crianças sobre a importância do amor no contexto da moralidade (Alves, Alencar, \& Ortega, 2012).

\section{Discussão}

Conforme se observou nos resultados, estudos na área da psicanálise e sexualidade abarcaram grande parte das produções científicas do país. Este dado não apresenta surpresas, uma vez que a psicanálise, com seu viés clínico, apresentou as primeiras teorias psicológicas sobre o amor, com os trabalhos de Freud (1910/1970, 1912/1970, 1918/1970), que analisa a gênese do sentimento de amor em sua relação com um objeto externo, no qual o amor forma-se pelo desejo libidinal; Reik (1944), que compreende o amor como algo diferente do desejo, expressando-se como um interesse apaixonado por uma outra personalidade, enquanto o desejo manifesta-se pelo interesse por um corpo enquanto objeto; e Erich Fromm (1956), que fundamenta o amor como uma arte, como uma atitude perante a vida.

As temáticas envolvendo o relacionamento conjugal também apresentaram grande incidência, uma vez que tal relacionamento perpassa diretamente o fenômeno do amor. De diferentes metodologias e perspectivas epistemológicas, os estudos nesta ênfase tentaram trazer respostas e abrindo novos questionamentos - sobre o estabelecimento, manutenção, qualidade, satisfação e término de relacionamentos, sendo este um dos pontos cruciais nos estudos deste campo.

Os estudos cujo público alvo foi a adolescência e juventude também se destacam, bem como sua relação com estudos sobre saúde e violência. As formas de relacionamentos amorosos atualmente têm cada vez mais se vinculado ao campo da sexualidade, o que pode, quando não devidamente trabalhado, pode causar sérias consequências, tais como DST's e gravidez indesejada. Buscar estratégicas efetivas de acesso à juventude é tarefa singular para o cuidado em saúde, bem como uma forma de conhecer as mudanças de valores e crenças, adaptar-se a elas, sem perder de vista a valorização do indivíduo e do cuidado consigo e com seu bem estar - tanto físico quanto psíquico.

A tecnologia também merece destaque enquanto nova forma de agenciamento amoroso na contemporaneidade. Tendo em vista que a internet tem revolucionado os meios de comunicação clássicos, influenciando diretamente nas relações interpessoais, os relacionamentos amorosos também têm sido alvo.

Sites de relacionamentos, chats de namoro e encontros virtuais são algumas das estratégias atuais no estabelecimento de novas relações amorosas, e a difusão da auto-imagem (nem sempre condizente com a realidade), permite, em muitos casos, maior interação dos indivíduos no ambiente virtual do que no não-virtual. Num estudo realizado por Markey e Welles (2002) estes chegaram a conclusão de que, num contexto experimental, indivíduos considerados introvertidos tendiam a ser moderadamente extrovertidos no espaço virtual, e indivíduos moderadamente extrovertidos tendiam aumentar seu nível de extroversão. Estes dados apontam também para a necessidade de maiores investigações, uma vez que tal ênfase tem despontado como um dos cenários de maior vinculação afetivo-sexual entre os jovens.

Além disso, no campo de produções acadêmicas, entre 2000 e 2010, além das publicações científicas em formato de artigos, livros de natureza científica que abordavam esta temática também se destacaram no território nacional, com ênfases em diversas correntes teóricas do campo psicológico, tais como as obras internacionais de David Buss (2000) em seu livro "Os Perigos da Paixão: Por que o Ciúme é tão Necessário quanto o Amor e o Sexo", e os de Helen Fisher, dentre eles: "Por que Amamos? A Natureza e a Química do Amor Romântico" (2006) e "Por Que Ele? Por Que Ela?" (2010). Em 2001, o pesquisador brasileiro Ailton Amélio Silva também publicou sua obra apresentando diversos dados científicos sobre o amor, em seu livro "O Mapa do Amor". Em 2009, ocorreu em Vitória o I Congresso Brasileiro de Pesquisa do Relacionamento Interpes- 
soal, tendo sua segunda edição ocorrida em 2011 e a terceira em 2013, proporcionando maior contato de estudantes ou pesquisadores com este tema.

Os resultados apresentaram também uma riqueza de perspectivas teóricas, metodologias utilizadas e fenômenos específicos dentro do campo de relacionamentos amorosos. Este dado corrobora com os apontamentos de Sternberg e Weiss (2006) ao afirmarem que uma única perspectiva teórica sobre o amor não seria capaz de abarcar todo o entendimento deste complexo fenômeno, sendo necessário portanto construir modelos que envolvem variáveis múltiplas (psicológicas, sociais, culturais e ambientais), justificando assim o grande número de pesquisas e teorias sobre o assunto.

\section{Considerações Finais}

Tendo em vista que o fenômeno do amor abarcando consigo os relacionamentos amorosos - desponta como um dos elementos centrais da cultura humana, inclusive do ciclo vital de nossa espécie, a compreensão deste complexo e multifacetado fenômeno desponta como de suma importância nos estudos e produções científicas, trazendo novas análises e perspectivas sobre o sucesso e fracasso nos relacionamentos.

Campo teórico já bastante expressivo nos Estados Unidos (Graham \& Christiansen, 2009), observou-se que ainda se encontra em consolidação no cenário nacional, com relativa expressividade a partir da primeira década do século XXI (de Andrade \& Wachelke, 2011), mas ainda mantendo um padrão relativamente pouco produtivo nesta temática. Mesmo que em crescente expansão, notou-se a carência de estudos no Brasil, principalmente estudos empíricos de cunho quantitativo e experimental - bastante presentes na literatura internacional. Contudo, apresenta diferentes modelos teóricos, epistemológicos e metodológicos, o que permite a constatação da fertilidade da temática e sua aplicação em diferentes naturezas, o que não exclui as divergências teóricas que elas mobilizam.

Nesta perspectiva, uma das sustentações que podem ser verificadas neste estudo é que, atualmente, não é possível um modelo comum de estudo sobre o fenômeno do amor. Ora complementares, ora divergentes, o grande número de teorias e pesquisas oferecem diferentes horizontes e explicações sobre o tema, o que também permite avaliá-la como um campo do conhecimento recente e em contínua construção. Esta construção faz-se de suma importância, uma vez que, mesmo bastante pesquisada internacionalmente e com desenvolvimento ainda incipiente no Brasil, ainda traz lacunas que necessitam de maiores sustentações e fundamentos teórico-metodológicos.

As principais teorias psicológicas sobre o amor não foram evidenciadas como substratos teóricos para fundamentar a temática do amor e relacionamentos amorosos em grande parte dos artigos nacionais, mas utiliza-se com maior frequência textos teóricos e/ou de cunho psicanalítico. Estas temáticas, mesmo possibilitando aportes teóricos importantes - e muitas vezes clássicos - nas discussões, trazem em muitos casos discussões mais sociológicas e/ou antropológicas frente ao fenômeno das relações amorosas, o que fragiliza a dimensão cognitiva, influenciando negativamente o fortalecimento de uma ciência psicologica do amor. Contudo, os resultados obtidos até o presente momento trazem contribuições importantes para uma compreensão cada vez mais aprimorada deste campo do conhecimento, através de pesquisas, sistematizações e rigor metodológico.

\section{Referências}

Alferes, V. R. (2004). Atração interpessoal, sexualidade e relações íntimas. In J. Vala \& M. B. Monteiro, Psicologia Social (6. ed.) Lisboa, Portugal: Fundação Calouste Gulbenkian.

Alves, A. D., Alencar, H. M., \& Ortega, A. C. (2012). Exemplos sobre a importância do amor: Estudo com crianças no contexto da moralidade. Temas em Psicologia, 20(1), 261-272.

Aron, A., Fisher, H., Mashek, D. J., Strong, G., Li, H., \& Brown, L. L. (2005). Reward, motivation, and emotion systems associated with early-stage intense romantic love. Journal of Neurophysiology, 94, 327-337. doi:10.1152/jn.00838.2004 
Baroncelli, L. (2011). Amor e ciúme na contemporaneidade: Reflexões psicossociológicas. Psicologia e Sociedade, 23(1), 163-170. doi:10.1590/ S0102-71822011000100018

Berscheid, E. (2006). Searching for the meaning of "love". In R. J. Sternberg \& K. Weis (Eds.), The new psychology of love (pp. 171-183). London: Yale University Press.

Berscheid, E., \& Hatfield, E. (1969). Interpersonal attraction. New York: Addison-Wesley.

Berti, M. P., Zilberman, M. L., Sophia, E. C., Gorenstein, C., Pereira, A. P., Lorena, A., ...Tavares, H. (2011). Validação de escalas para avaliação do amor patológico. Revista de Psiquiatria Clínica, 38(4), 135-138. doi:10.1590/S010160832011000400004

Bezerra, P. V., \& Justo, J. S. (2010). Relacionamentos amorosos na Pós-Modernidade: Análise de consultas apresentadas em sites de agenciamento amoroso. Pesquisas e Práticas Psicossociais, 4(2), 193-204.

Bressaneli, J., \& Teixeira, A. M. R. (2012). Erotomania: Os impasses do amor e uma resposta psicótica. Ágora: Estudos em Teoria Psicanalitica, 15, 437-451. doi:10.1590/S151614982012000300006

Buss, D. M. (2000). Os perigos da paixão: Por que o ciúme é tão necessário quanto o amor e o sexo (M. Campello, Trad.). Rio de Janeiro, RJ: Objetiva.

Buss, D. M. (2006). The evolution of love. In R. J. Sternberg \& K. Weis (Eds.), The new psychology of love (pp. 65-86). London: Yale University Pres.

Bystronski, B. (1992). A liberação dos costumes $e$ suas consequências sobre os relacionamentos amorosos heterossexuais (Dissertação de mestrado não-publicada, Instituto de Filosofia e Ciências Humanas, Universidade Federal do Rio Grande do Sul, Porto Alegre, RS, Brasil).

Bystronski, B. (1995). Teorias e processos psicossociais da intimidade interpessoal. In A. Rodrigues, Psicologia Social para principiantes: Estudo da interação humana (pp. 86-126). Petrópolis, RJ: Vozes.

Camargo, B. V., Giacomozzi, A. I., Wachelke, J. F. R., \& de Aguiar, A. (2010). Relações amorosas, comportamento sexual e vulnerabilidade de adolescentes afrodescendentes e brancos em relação ao HIV/aids. Saúde e Sociedade, 19(Supl. 2), 36-50. doi:10.1590/S0104-12902010000600005

Cassepp-Borges, V., \& Pasquali, L. (2011). Características psicométricas da Relationship Assessment Scale. Psico-USF, 16(3), 255-264. doi:10.1590/S1413-82712011000300002

Cassepp-Borges, V., \& Pasquali, L. (2012). Estudo nacional dos atributos psicométricos da Escala Triangular do Amor de Sternberg. Paidéia (Ribeirão Preto), 22(51), 21-31. doi:10.1590/ S0103-863X2012000100004

Cassepp-Borges,V., \& Teodoro, M. L. M. (2007). Propriedades psicométricas da versão brasileira da Escala Triangular do Amor de Sternberg. Psicologia: Reflexão e Crítica, 20(3), 513-522. doi:10.1590/S0102-79722007000300020

Cassepp-Borges, V., \& Teodoro, M. L. M. (2009). Versíon reducida de la Escala Triangular del Amor: características del sentimiento em Brasil. Revista Interamericana de Psicologia, 43(1), 30-38.

Centeville, V., \& de Almeida, T. (2007). Ciúme romântico e a sua relação com a violência. Psicologia em Revista 16(1-2), 71-89.

Chaves, J. C. (2010). As percepções de jovens sobre os relacionamentos amorosos na atualidade. Psicologia em Revista, 16(1), 28-46. doi:10.5752/ P.1678-9563.2010V16N1P28

Cooper, V., \& Pinto, B. (2008). Actitudes ante el amor y la teoría de Sternberg. Un estúdio correlacional em jovenes universitarios de 18 a 24 años de edad. Ajayu, 6(2), 181-206.

Costa, V., \& Fernandes, S. C. S. (2012). O que pensam os adolescentes sobre o amor e o sexo? Um estudo na perspectiva das representações sociais. Psicologia \& Sociedade, 24(2), 391-401. doi:10.1590/S0102-71822012000200017

De Almeida, T. (2012). O ciúme romântico atua como uma profecia autorrealizadora da infidelidade amorosa? Estudos de Psicologia (Campinas), 29(4), 489-498. doi:10.1590/S0103$-166 \times 2012000400004$

De Andrade, A. L., \& Garcia, A. (2009). Atitudes e crenças sobre o amor: Versão brasileira da Escala de Estilos de Amor. Interpersona: An International Journal on Personal Relationships, 3, 89-102.

De Andrade, A. L., \& Garcia, A. (2012). Desenvolvimento de uma medida multidimensional para 
avaliação de qualidade em relacionamentos românticos - Aquarela-R. Psicologia: Reflexão e Crítica, 25(4), 634-643. doi:10.1590/S010279722012000400002

De Andrade, A. L., Garcia, A., \& Cano, D. S. (2009). Preditores da satisfação global em relacionamentos românticos. Psicologia: Teoria e Prática, 11(3), 143-156.

De Andrade, A. L., Garcia, A., \& Cassepp-Borges, V. (2013). Evidências de validade da Escala Triangular do Amor de Sternberg - Reduzida (ETAS-R). Psico-USF, 18(3), 501-510. doi:10.1590/ S1413-82712013000300016

De Andrade, A. L., Sánchez-Aragón, R., \& Wachelke, J. F. R. (2007). Validade fatorial da Escala de Estilos de Amor [CD]. In Anais da XXXVII Reunião Anual de Psicologia da Sociedade Brasileira de Psicologia. Florianópolis, SC: Sociedade Brasileira de Psicologia.

De Andrade, A. L., \& Wachelke, J. (2011). The association of structural configurations of romantic relationships with beliefs about couple relationships: A social representations study. Anales de Psicología, 27, 834-842.

De Araújo, L. M. (2012). Amor e violência: Um paradoxo das relações de namoro e do 'ficar' entre jovens brasileiros. Ciência \& Saúde, 17(9), 25532554. doi:10.1590/S1413-81232012000900035

Dela Coleta, A. S. M., Dela Coleta, M. F., \& Guimarães, J. L. (2008). O amor pode ser virtual? O relacionamento amoroso pela internet. Psicologia em Estudo, 13(2), 277-285.

Dela Coleta, M. F. (1989). A medida da satisfação conjugal: Adaptação de uma escala. Psico, 18(2), 90-112.

Dela Coleta, M. F. (1990). Satisfacción marital: estudios con sujetos brasileños. Revista de Psicologia Social y Personalidad, 6(1), 101-116.

Dela Coleta, M. F. (1991). Causas atribuídas ao sucesso e fracasso no casamento. Psico, 22(2), 2139.

Dias, A. R. C., \& Machado, C. (2011). Amor e violência na intimidade: Da essência à construção social. Psicologia e Sociedade, 23(3), 496-505. doi:10.1590/S0102-71822011000300007

Donnamaria, C. P., \& Terzis, A. (2009). O amor caiu na rede: Sobre a procura de parceiro e a evolução de vínculos amorosos na Internet. Revista SPAGESP, 10(2), 45-49.
Doron, R., \& Parot, F. (1991). Dictionnaire de Psychologie. Paris: Presses Universitaires de France.

Duarte, J. P., \& Rocha-Coutinho, M. L. (2011). "Namorido": Uma forma contemporânea de conjugalidade? Psicologia Clínica, 23(2), 117-135. doi:10.1590/S0103-56652011000200008

Engel, G., Olson, K. R., \& Patrick, C. (2002). The personality of love: Fundamental motives and traits related to components of love. Personality and Individual Differences, 32(5), 839-853. doi:10.1016/S0191-8869(01)00090-3

Ferrari, I. F. (2009). Acerca do amor e algumas de suas particularidades na psicose. Arquivos Brasileiros de Psicologia, 61(3), 87-96.

Fisher, H. (2006). Por que amamos: A natureza e a química do amor romântico. Rio de Janeiro, RJ: Record.

Fisher, H. (2010). Por que Ele? Por que Ela? Como encontrar o amor verdadeiro conhecendo seu tipo de personalidade. Rio de Janeiro, RJ: Rocco.

Freud, S. (1970). Um tipo especial de escolha de objeto deita pelos homens (Contribuições à psicologia do amor I). In J. Strachey (Ed.), Edição standard brasileira das obras psicológicas completas de Sigmund Freud (J. Salomão, Trad., Vol. 11, pp. 147-158). Rio de Janeiro, RJ: Imago. (Original publicado em 1910)

Freud, S. (1970). Sobre a tendência universal à depreciação na esfera do amor (Contribuições à psicologia do amor II). In J. Strachey (Ed.), Edição standard brasileira das obras psicológicas completas de Sigmund Freud (J. Salomão, Trad., Vol. 11, pp. 159-174). Rio de Janeiro, RJ: Imago. (Original publicado em 1912)

Freud, S. (1970). O tabu da virgindade (Contribuições à psicologia do amor III). In J. Strachey (Ed.), Edição standard brasileira das obras psicológicas completas de Sigmund Freud (J. Salomão, Trad., Vol. 11, pp. 175-192). Rio de Janeiro, RJ: Imago. (Original publicado em 1918)

Freud, S. (1973). Cinco lições de psicanálise e contribuições à psicologia do amor. Rio de Janeiro, RJ: Imago.

Fromm, E. (1956). A arte de amar. Rio de Janeiro, RJ: Zahar.

Gianfaldoni, M. H. T. A., Teixeira, R. R. P., Comolatti, C., Pareto, C., Careli, G., Figueiredo, G., 
\& Lopez, L. (2009). Critérios de seleção para parceiros de curto e de longo prazo de universitárias paulistas. Psicologia em Revista, 18(1), 99-111.

Gouveia, V., de Carvalho, E. A. B., Santos, F. A. dos, \& de Almeida, M. R. (2013). Escala Tetrangular do Amor: Testando sua estrutura e invariância fatorial. Psicologia: Ciência e Profissão, 33(1), 32-45. doi:10.1590/S1414-98932013000100004

Gouveia, V. V., Fonseca, P. N. da, Cavalcanti, J. P. N., Diniz, P. K. da C., \& Dória, L. C. (2009). Versão abreviada da Escala Triangular do Amor: Evidências de validade fatorial e consistência interna. Estudos de Psicologia (Natal), 14(1), 3139. doi:10.1590/S1413-294X2009000100005

Graham, J. M., \& Christiansen, K. (2009). The reliability of romantic love: A reliability generalization meta-analysis. Personal Relationships, 16 , 49-66. doi:10.1111/j.1475-6811.2009.01209.x

Guede, D. D., Monteiro-Leitner, J., \& Machado, K. C. R. (2008). Rompimento amoroso, depressão e auto-estima: Estudo de caso. Revista Mal-Estar da Subjetividade, 8(3), 603-643.

Hazan, C., \& Shaver, P. (1987). Romantic love conceptualized as an attachment process. Journal of Personalityand Social Psychology, 52, 511-524.

Hendrick, C., \& Hendrick, S. S. (2006). Styles of romantic love. In R. J. Sternberg \& K. Weis (Eds.), The new psychology of love (pp. 149-170). London: Yale University Press.

Hendrick, C., Hendrick, S. S., \& Dicke, A. (1988). The Love Attitudes Scale: Short form. Journal of Social and Personality Relationships, 15, 147-159.

Hernandez, J. A. E. (1999). Validação da estrutura da Escala Triangular do Amor: Análise fatorial confirmatória. Aletheia, 9, 15-25.

Hernandez, J. A. E., \& Biasetto, I. M. (2003). Os componentes do amor e a satisfação. Psicologia: Ciência e Profissão, 21(3), 58-69.

Hernandez, J. A. E., \& Roveda, A. M. A. (1998). Auto-estima e amor. Aletheia, 8, 117-133.

Kanemasa, Y., Taniguchi, J., \& Daibo, I. (2004). Love styles and romantic love experiences. Social Behavior and Personality, 32, 265-282.

Kenrick, D. T. (2006). A dynamical evolutionary of view of love. In R. J. Sternberg \& K. Weis (Eds.), The new psychology of love (pp. 15-34). London: Yale University Press.
Lakatos, E. M., \& Marconi, M. A. (2003). Fundamentos de metodologia científica (5. ed.). São Paulo, SP: Atlas.

Lee, J. A. (1973). Colours of love: An exploration of the ways of loving. Toronto, Canada: New Press.

Lee, J. A. (1977). A typology of stiles of loving. Personality and Social Psycholoy Bulletin, 3, 173182.

Levy, L., \& Gomes, I. C. (2008). Relação conjugal, violência psicológica e complementaridade fusional. Psicologia Clínica, 20(2), 163-172. doi:10.1590/S0103-56652008000200012

Manuel, S. (2009). Presentes perigosos: Dinâmicas de risco de infecção ao HIV/Aids nos relacionamentos de namoro em Maputo. Physis: Revista de Saúde Coletiva, 19(2), 371-386. doi:10.1590/ S0103-73312009000200007

Marcondes, M. V., Trierweiler, M., \& Cruz, R. M. (2006). Sentimentos predominantes após o término de um relacionamento amoroso. Psicologia: Ciência e Profissão, 26(1), 94-105. doi:10.1590/S1414-98932006000100009

Markey, P. M., \& Wells, S. M. (2002). Interpersonal perception in internet chat rooms. Journal of Research in Personality, 36, 134-146. doi:10.1006/ jrpe. 2002.2340

Martins-Silva, P. de O., Trindade, Z. A., \& Silva, A. da, Jr. (2013). Teorias sobre o amor no campo da Psicologia Social. Psicologia: Ciência e profissão, 33(1), 16-31. doi:10.1590/S141498932013000100003

Maslow, A. (1974). Introdução à Psicologia do Ser. Rio de Janeiro, RJ: Eldorado.

Matos, M., Féres-Carneiro, T., \& Jablonski, B. (2005). Adolescência e relações amorosas: Um estudo sobre jovens das camadas populares cariocas. Interação em Psicologia, 9(1), 21-33.

May, S. (2012). Amor: Uma história. Rio de Janeiro, RJ: Zahar.

Menandro, P. R. M., Rolke, R. K., \& Bertollo, M. (2005). Concepções sobre relações amorosas / conjugais e sobre seus protagonistas: Um estudo com provérbios. Psicologia Clinica, 17(2), 81100 .

Mônego, B. G., \& Teodoro, M. L. M. (2011). A teoria triangular do amor de Sternberg e o modelo dos cinco grandes fatores. Psico-USF, 16(1), 97105. doi:10.1590/S1413-82712011000100011 
Mosmann, C. P., Lomando, E., \& Wagner, A. (2010). Coesão e adaptabilidade conjugal em homens e mulheres hetero e homossexuais. Barbaroi, 33, $135-152$.

Mosmann, C., Wagner, A., \& Féres-Carneiro, T. (2006). Qualidade conjugal: Mapeando conceitos. Paidéia (Ribeirão Preto), 16, 315-325. doi:10.1590/S0103-863X2006000300003

Moura, L. B. A., Lefevre, F., \& Moura, V. (2012). Narrativas de violências praticadas por parceiros íntimos contra mulheres. Ciência \& Saúde Coletiva, 17(4), 1025-1035. doi:10.1590/S141381232012000400024

Reik, T. (1944). A psychologist looks at love. New York: Farrar \& Rinehart.

Reis, B. F. (1992). O amor à luz da Psicologia Científica. Psicologia: Reflexão \& Crítica, 5(2), 2340.

Reis, R. K., \& Gir, E. (2010). Convivendo com a diferença: $O$ impacto da sorodiscordância na vida afetivo-sexual de portadores do HIV/AIDS. Revista da Escola de Enfermagem, 44(3), 759 765. doi:10.1590/S0080-62342010000300030

Rubin, Z. (1970). Measurement of romantic love. Journal of Personality and Social Psychology, 16(2), 265-273. doi:10.1037/h0029841

Russo, G. (2011). Amor e dinheiro: Uma relação possível? Caderno CRH, 24(61), 121-134. doi:10.1590/S0103-49792011000100009

Sánchez-Aragón, R. (2005). Mexican love styles. In A. Garcia (Ed.), Personal relationships: International studies (pp. 64-77). Vitória, ES: Núcleo Interdisciplinar para o Estudo do Relacionamento Interpessoal, Universidade Federal do Espírito Santo.

Schlösser, A., Dalfovo, D., \& Delvan, J. S. (2012). Um estudo sobre o amor: Diálogos entre Sigmund Freud e Erich Fromm. Psicologia Argumento, 30(70), 567-573. doi:10.7213/psicol. argum. 6143

Scorsolini-Comin, F., \& Santos, M. A. dos. (2010). Satisfação conjugal: Revisão integrativa da literatura científica nacional. Psicologia: Teoria e Pesquisa, 26(3), 525-532. doi:10.1590/S010237722010000300015

Silva, A. A. (2001). O mapa do amor. São Paulo, SP: Gente.

Skinner, B. F. (1991). Questões recentes da análise do comportamento. Campinas, SP: Papirus.
Sophia, E. C., Tavares, H., \& Ziberman, M. L. (2007). Amor patológico: Um novo transtorno psiquiátrico? Revista Brasileira de Psiquiatria, 29(1), 55-62.

Sternberg, R. J. (1986). A triangular theory of love. Psychological Review, 93, 119-135. doi:10.1037/0033-295X.93.2.119

Sternberg, R. J. (1989). El triangulo del amor: intimidad, passión y compromisso. Barcelona, España: Paidós.

Sternberg, R. J. (2000). La experiência del amor. Barcelona, España: Paidós.

Sternberg, R. J., \& Barnes, M. L. (1985). Real and ideal others in romantic relationship: Is for a crowd? Journal of Personality and Social Psychology, 4, 1586-1608.

Sternberg, R. J., \& Grajek, S. (1984). The nature of love. Journal of Personality and Social Psychology, 47, 312-329.

Sternberg, R. J., \& Weis, K. (2006). The new psychology of love. London: Yale UniversityPress.

Vendrame, T., Costa, A. L. da, Sanches, C. C., Gulassa, D. C. R., Sophia, E. C., \& Zilberman, M. L. (2012). Impulsiveness in patients with pathological love. Jornal Brasileiro de Psiquiatria, 61(3), 191-192. doi:10.1590/S004720852012000300013

Villa, M. B., Del Prette, Z. A. P., \& Del Prette, A. (2007). Habilidades sociais conjugais e filiação religiosa: Um estudo descritivo. Psicologia em Estudo, 12(1), 23-32.

Wachelke, J. F. R., de Andrade, A. L., Cruz, R. M., Faggiani, R. B., \& Natividade, J. C. (2004). Medida da satisfação em relacionamento de casal. Psico-USF, 9(1), 11-18.

Wachelke, J. R., de Andrade, A. L., Souza, A. M., \& Cruz, R. M. (2007). Estudo complementar da validade fatorial da Escala Fatorial de Satisfação em Relacionamento e predição de satisfação global com a relação. Psico-USF, 12(2), 221-225.

Wilson, G. (1981). The Coolidge effect: An evolutionary account of human sexuality. New York:Willian Morrow. 